\title{
Second generation monolithic full-depletion radiation sensor with integrated CMOS circuitry
}

\author{
J. D. Segal, C. J. Kenney, S.I. Parker, C. H. Aw, W. J. Snoeys, B. Wooley, J. D. Plummer
}

\begin{abstract}
A second-generation monolithic silicon radiation sensor has been built and characterized. This pixel detector has CMOS circuitry fabricated directly in the high-resistivity floatzone substrate. The bulk is fully depleted from bias applied to the backside diode. Within the array, PMOS pixel circuitry forms the first stage amplifiers. Full CMOS circuitry implementing further amplification as well as column and row logic is located in the periphery of the pixel array. This allows a sparse-field readout scheme where only pixels with signals above a certain threshold are readout. We describe the fabrication process, circuit design, system performance, and results of gamma-ray radiation tests.
\end{abstract}

\section{INTRODUCTION}

Integrated monolithic silicon pixel detectors for particle physics and $\mathrm{x}$-ray applications have several advantages, including low collection electrode capacitance and good spatial resolution. Previously, Snoeys reported on the first generation monolithic bulk pixel detector [1], and beam test results were reported in [2]. In this paper, we report results for the second generation sensor, with increased area and resolution, improved yield, and a more sophisticated circuit taking advantage of the integrated CMOS capabilities.

\section{A. Sensor Structure Overview}

Fig. 1 illustrates some of the key features of our detector. We start with $300 \mu \mathrm{m}$ thick high-resistivity p-type silicon wafers. During operation, the bulk is fully depleted from an ndiffusion on the backside. Within the pixel array, each pixel consists of a small $\mathrm{p}+$ collection electrode surrounded by an nwell containing PMOS pre-amplifier circuitry. Full CMOS row and column circuitry and control logic are outside the array. The n-wells and p-wells containing the CMOS devices are implanted directly into the bulk silicon, which also supports the PIN diodes.

\section{B. High Voltage termination structure}

This structure requires double-sided processing, which is challenging because the backside of the wafer is placed on a variety of surfaces that can result in scratches and other mechanical damage. To reduce this risk, a simpler and more robust high voltage terminations structure was developed

Manuscript received November 13, 2010. This work was supported in part by the DARPA Computational Prototyping Project, in part by the U.S. Department of Energy, and in part by the Texas National Research Laboratory Commission

J. D. Segal and C. J. Kenney are with SLAC, Menlo Park, CA 94025 (email: jsegal@slac.stanford.edu)

S. I. Parker is with University of Hawaii

C. H. Aw is with UOB Venture Management, Singapore

W. J. Snoeys is with CERN

B. Wooley and J. Plummer are with Stanford University

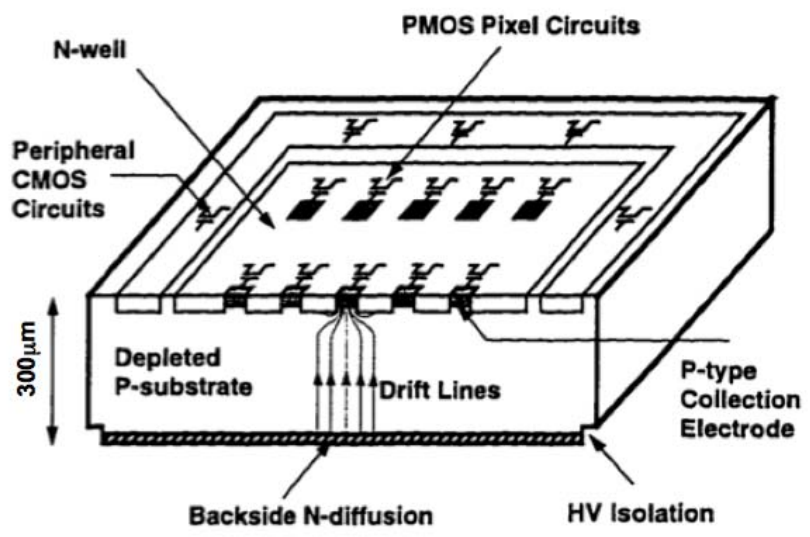

Fig. 1. Schematic illustration of the integrated sensor structure

using a vertical etch to define the edge of the backside diode

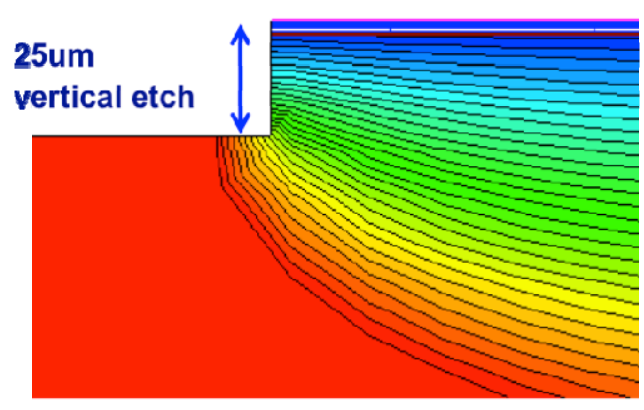

Fig. 2. Simulated equipotential contours for the vertical high voltage termination structure

[3]. The structure is illustrated in Fig. 2, which shows simulated equipotentials under bias. The vertical cut eliminates the curvature at the edge of the diffused diode that causes enhanced electric fields. After the vertical etch, the vertical plane must be passivated by subsequent thermal oxidation to prevent surface leakage, and in the case of p-type silicon, implanted with a boron surface implant.

This structure requires only one backside mask instead of three needed for the floating ring termination structure used in the previous generation [1]. The front side of the wafer, with more complex patterns and smaller geometries, is therefore subject to less damage, resulting in improved yield. Furthermore, this simplified backside structure is more robust itself, resulting in reduced yield loss from high-voltage diode shorts [3]. 


\begin{tabular}{|c|c|c|c|}
\hline & MASK\# & PROCESS STEP & PARAMETERS \\
\hline 1 & & starting material & $<100>$ p-type $12 \mathrm{~K}$-ohm wafers \\
\hline 2 & & initial oxidation & $1 \mathrm{hr} 1000 \mathrm{C}$ wet \\
\hline 3 & & backside initial oxide strip & wet etch \\
\hline 4 & & backside $\mathrm{n}$-implant & phosphorus $100 \mathrm{keV} 2 \mathrm{E} 14 / \mathrm{cm} 2$ \\
\hline 5 & & initial oxidation \#2 & $1 \mathrm{hr} 1000 \mathrm{C}$ wet \\
\hline 6 & MASK 1 & $\mathrm{~N}$-well mask (topside) and etch & wet etch \\
\hline 7 & & $\mathrm{~N}$-well implant & phosphorus $100 \mathrm{keV} 2.5 \mathrm{E} 12 / \mathrm{cm} 2$ \\
\hline 8 & MASK 2 & P-well mask (topside) and etch & wet etch \\
\hline 9 & & P-well implant & boron $100 \mathrm{keV} 3 \mathrm{E} 12 / \mathrm{cm} 2$ \\
\hline 10 & & Well drive & $30 \mathrm{~min} 1000 \mathrm{C}$ wet, $16 \mathrm{hrs} 1150 \mathrm{C}$ inert \\
\hline 11 & & topside oxdie strip & wet etch \\
\hline 12 & & thin buffer oxidation & $42 \mathrm{~min} 950 \mathrm{C}$ dry \\
\hline 13 & & nitride deposition & $800 \mathrm{~A}$ front and back \\
\hline 14 & MASK 3 & field oxide mask and nitride etch & dry etch \\
\hline 15 & MASK 4 & field implant mask & boron $100 \mathrm{keV} 3 \mathrm{E} 13 / \mathrm{cm} 2$ \\
\hline 16 & & field oxidation & $3 \mathrm{hr} 10 \mathrm{~min} 1000 \mathrm{C}$ wet \\
\hline 17 & & nitride strip & wet etch \\
\hline 18 & & buffer oxide strip & short wet etch (leave field oxide) \\
\hline 19 & & thin clean-up oxide & $14.5 \mathrm{~min} 850 \mathrm{C}$ wet \\
\hline 20 & MASK 5 & NMOS threshold adjust implant & boron $35 \mathrm{keV} 1 \mathrm{e} 12 / \mathrm{cm} 2$ \\
\hline 21 & MASK 6 & PMOS threshold adjust implant & arsenic $80 \mathrm{keV} 1 \mathrm{e} 12$ \\
\hline 22 & & thin oxide strip & short wet etch (leave field oxide) \\
\hline 23 & & gate oxidation & $14.5 \mathrm{~min} 850 \mathrm{C}$ wet \\
\hline 24 & MASK 7 & buried contact mask and etch & short wet etch \\
\hline 25 & & backside oxide strip & wet etch \\
\hline 26 & & polysilicon deposition & $1500 \mathrm{~A}$ front and back \\
\hline 27 & & nitride deposition & $300 \mathrm{~A}$ front and back \\
\hline 28 & & backside $\mathrm{n}+$ implant & phosphorus $100 \mathrm{keV} 1 \mathrm{E} 16 / \mathrm{cm} 2$ \\
\hline 29 & MASK 8 & backside diode mask and etch & 25 um dry etch \\
\hline 30 & & smoothing trench oxidation & $10 \mathrm{~min} 1000 \mathrm{C}$ wet \\
\hline 31 & & backside oxide strip & wet etch \\
\hline 32 & & backside trench passivation & $10 \mathrm{~min} 1000 \mathrm{C}$ wet \\
\hline 33 & & backside field implant & $\begin{array}{l}\text { boron } 100 \mathrm{keV} 6 \mathrm{E} 11 / \mathrm{cm} 2 \text { at } 15 \mathrm{deg}, \\
\text { implant } 4 \mathrm{X} \text {, once from each direction }\end{array}$ \\
\hline 34 & MASK 9 & polysilicon mask and etch & dry etch through nitride and poly \\
\hline 35 & & polysilicon sidewall oxidation & $6 \mathrm{~min} 850 \mathrm{C}$ wet \\
\hline 36 & MASK 10 & $n+$ implant & arsenic $80 \mathrm{keV} 1 \mathrm{e} 16$ \\
\hline 37 & & anneal/getter 1 & $30 \mathrm{~min} 1000 \mathrm{C}$ inert \\
\hline 38 & MASK 11 & $p+$ implant & boron $15 \mathrm{keV} 2 \mathrm{e} 15 / \mathrm{cm} 2$ \\
\hline 39 & & anneal/getter 2 & $35 \mathrm{~min} 900 \mathrm{C}$ inert \\
\hline 40 & & nitride strip & wet etch, front and back \\
\hline 41 & & selective tungsten deposition & front and back \\
\hline 42 & & LTO deposition & 5000A oxide, front and back \\
\hline 43 & MASK 12 & contact mask and etch & dry etch \\
\hline 44 & & backside LTO removal & dry etch, leave trench passivation \\
\hline 45 & & metal 1 deposition & $5000 \mathrm{~A} \mathrm{Al} / \mathrm{Si}$ \\
\hline 46 & MASK 13 & metal 1 mask and etch & dry etch \\
\hline 47 & & LTO deposition & 2000A undoped oxide +5500 doped \\
\hline 48 & MASK 14 & via mask and etch & dry etch through nitride and poly \\
\hline 49 & & metal 2 depostion & 1 um Al \\
\hline 50 & MASK 15 & metal 2 mask and etch & dry etch \\
\hline
\end{tabular}

Fig. 3. Sensor process flow details

\section{SENSOR PROCESS}

The starting material is high resistivity float-zone p-type silicon. Twin wells are implanted and diffused directly into the bulk to support a $2 \mu \mathrm{m}$ CMOS process with double-layer metal interconnects. The process includes 15 masking steps, including one backside mask.

The backside mask (Mask \#8) defines a $25 \mu \mathrm{m}$ vertical etch used to terminate the high-voltage junction. After the backside etch step, sacrificial oxidation is performed to smooth and clean the etched surface, followed by a second oxidation to passivate the surface. A shallow boron implant into the vertical surface is also required, and must be performed four times, once from each direction, to implant the four vertical faces.

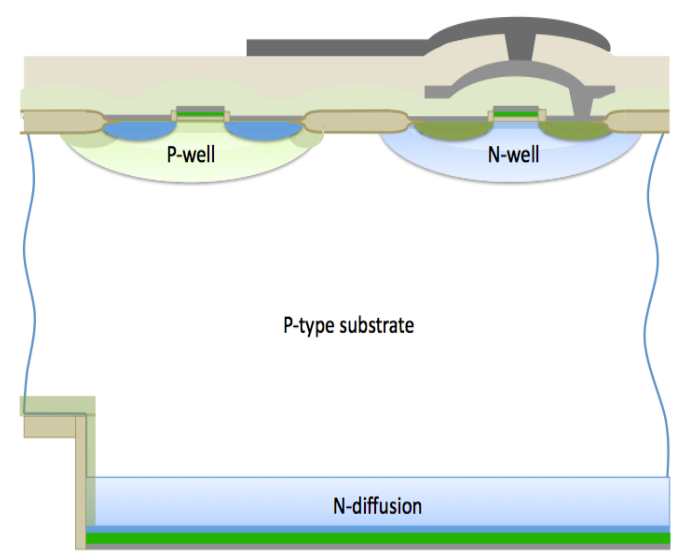

Fig. 4. Cross section of integrated sensor process

After the backside diode process is mostly completed, the backside surface, which comes into contact with equipment, is covered with either nitride or tungsten to prevent mechanical damage during subsequent processing.

Full details of the process are listed in Fig. 3. The final cross-section is illustrated in Fig 4. All processing was done at the Stanford Nanofabrication Facility (SNF).

\section{A. Bulk Leakage Results}

Backside leakage for the $29 \mathrm{~mm}^{2}$ sensor ranged from $10 \mathrm{nA}$ to $1 \mu \mathrm{A}$. This measurement includes the diode perimeter current that constitutes the majority of the leakage current and does not affect sensor performance because it is collected by a guard ring. Leakage in the pixel array only, not including perimeter leakage, was estimated to be approximately $150 \mathrm{nA} / \mathrm{cm}^{3}$, corresponding to a lifetime of $15 \mathrm{~ms}$.

\section{B. High Voltage Diode Results}

Full depletion occurred at $40 \mathrm{~V}$ to $55 \mathrm{~V}$ applied to the backside diode. The backside diode is subject to mechanical damage during processing and high electric fields under bias. Nevertheless, the yield loss from backside diode shorts was less than $8 \%(0 / 12$ detectors fail $)$.

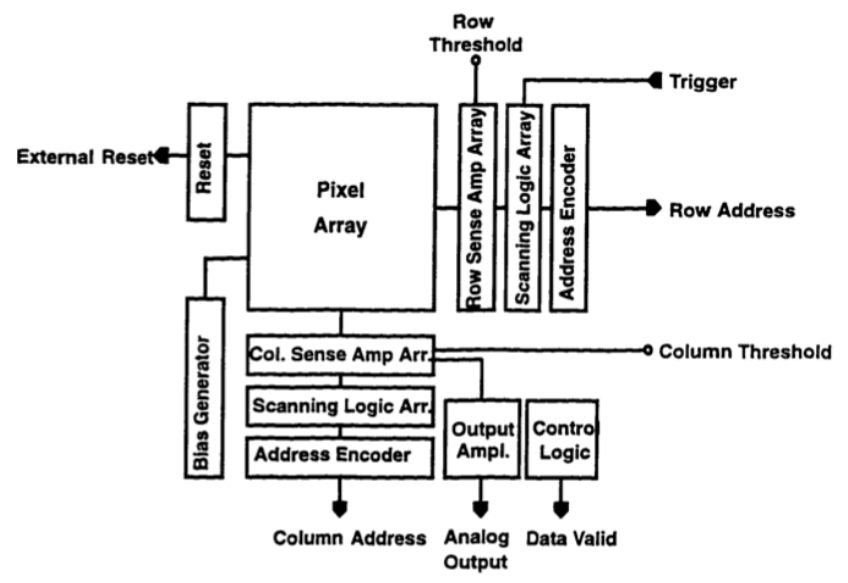

Fig. 5. Sensor Circuit Architecture 


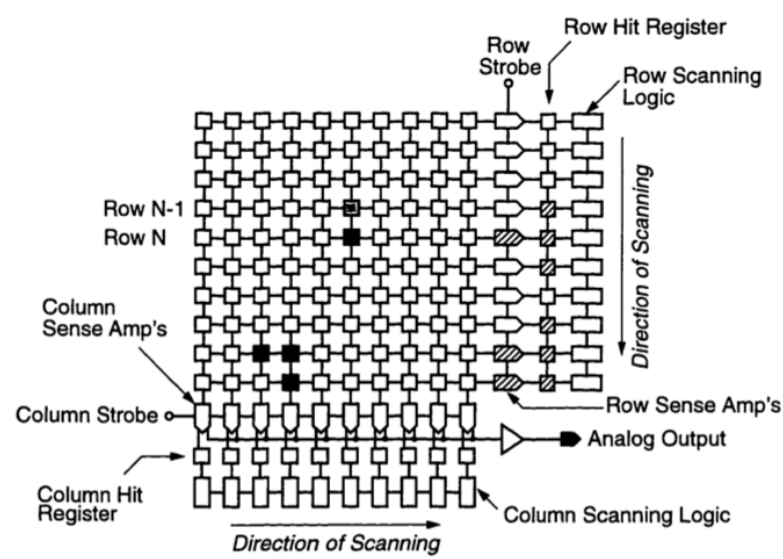

Fig. 6. Sparse-field readout

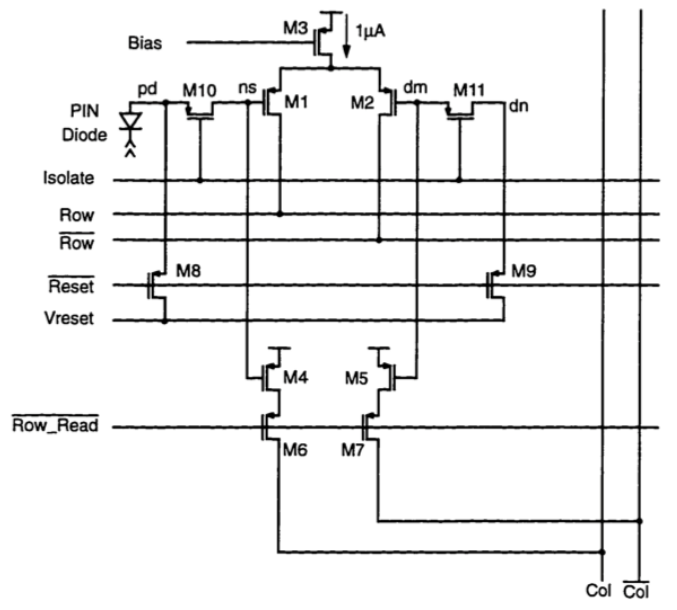

Fig. 7. Pixel Circuit

\section{CIRCUIT}

The circuit architecture is shown in Fig. 5 [4]. The array contains 32 by 32 pixels.

Sparse-field read-out operation is illustrated in figure 6 . When the detector is externally triggered for read-out, the row scanning logic scans the rows to determine which ones have been hit, and activates those rows. The column scanning logic then scans the active rows to determine which pixels on those rows have been hit, and reads out their analog pulse heights.

PMOS pixel circuitry provides the first stage amplification of the signal. Each pixel is $65 \mu \mathrm{m}$ by $67 \mu \mathrm{m}$. A schematic of the pixel circuit is shown in Fig. 7.

\section{A. Circuit test results}

Noise measurements are shown in Fig. 8, with a standard deviation of $1.6 \mathrm{keV}$. Since a minimum ionizing particle traversing $300 \mu \mathrm{m}$ of silicon deposits $86 \mathrm{keV}$ (most probable value), the signal-to-noise ratio would be 54:1 for a particle physics application.

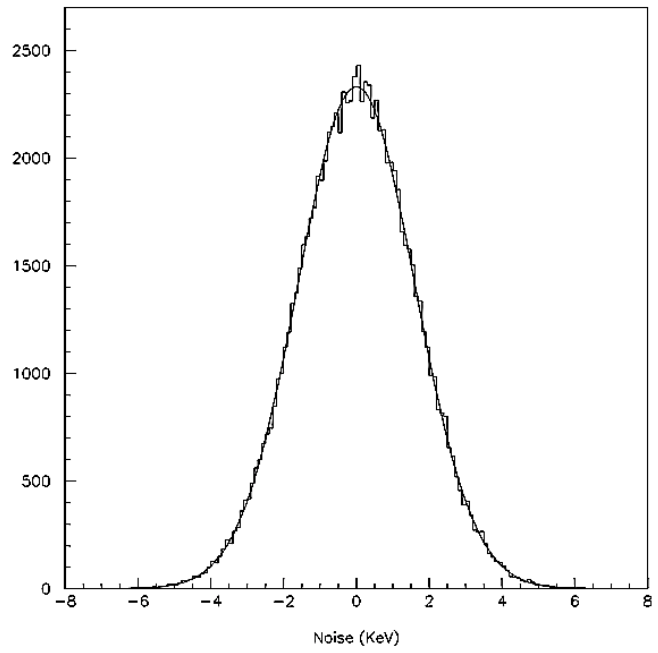

Fig. 8. Measured pixel noise with $\sigma=1.6 \mathrm{keV}$
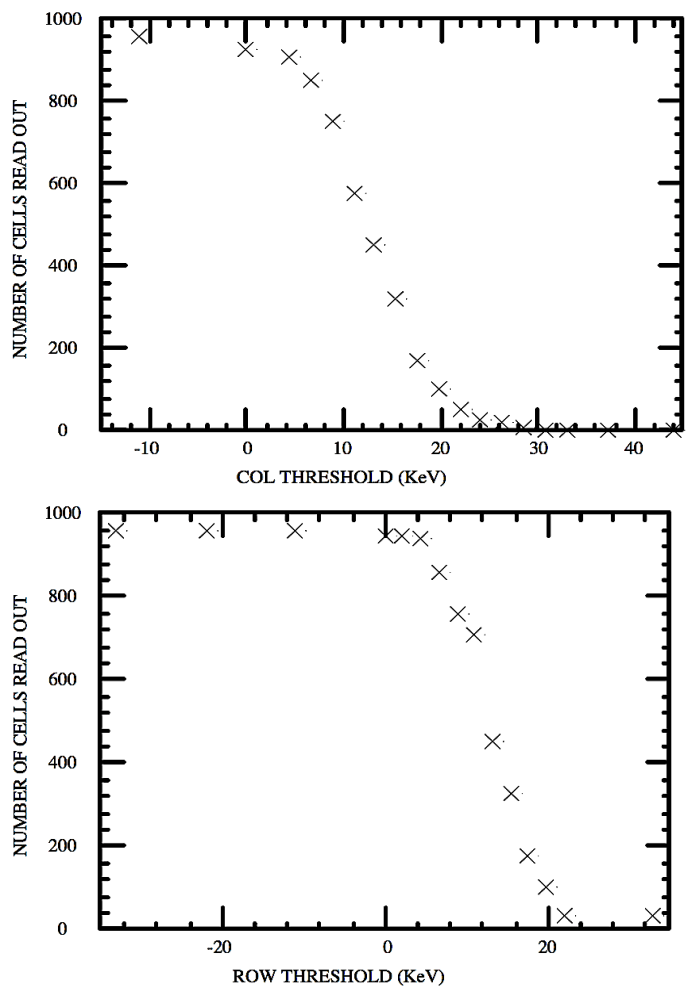

Fig. 9. Row and column threshold distributions

The row and column thresholds are shown in Fig. 9. Comparing the threshold spread to the signal from a minimum ionizing particle gives a measure of the efficiency of the detector in sparse field mode, or the chance that a real event will trigger the read-out. The worst case, or smallest, pixel signal for a minimum ionizing particle is $21 \mathrm{keV}$, assuming that the charge is divided between four pixels. Both the row and column threshold spread, at $16 \mathrm{keV}$ and $20 \mathrm{keV}$ respectively, are low enough to expect close to $100 \%$ efficiency. 


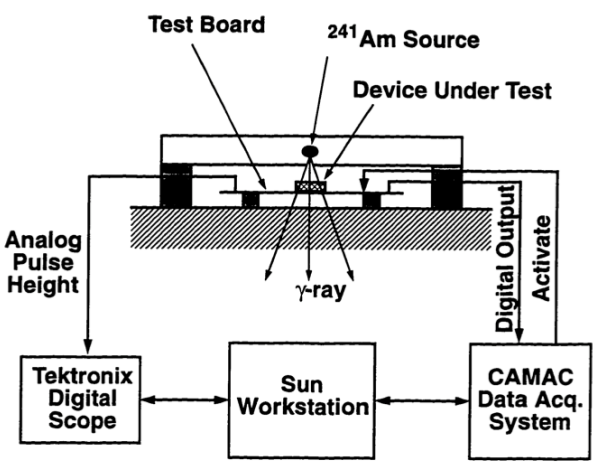

Fig. 10. Test set-up for $\gamma$-ray radiation tests
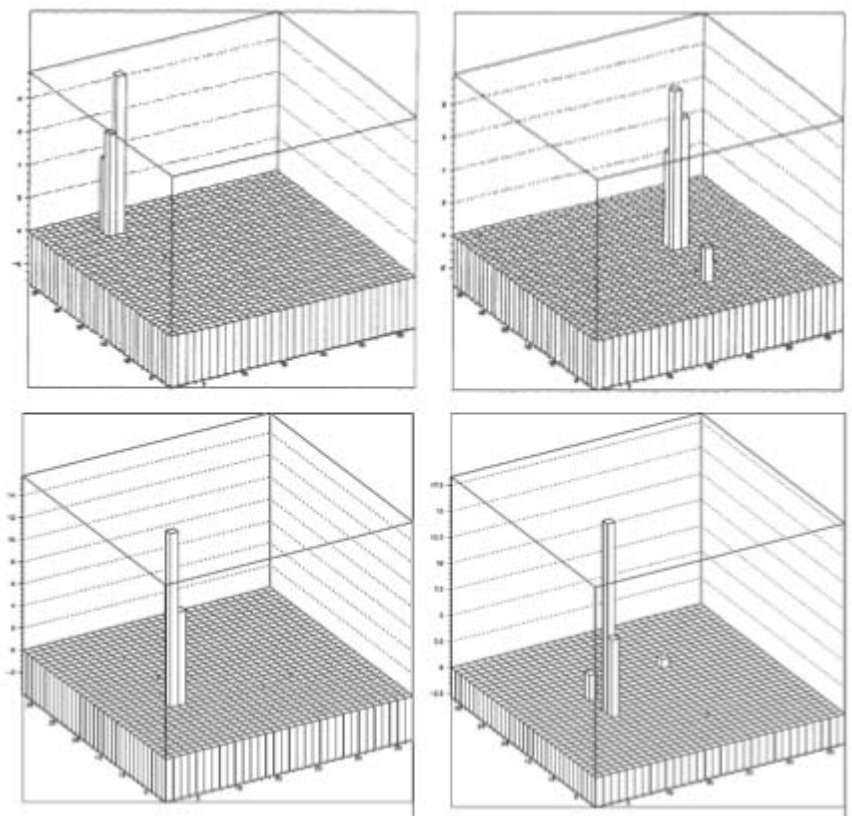

Fig. 11. Examples of single event $\gamma$-ray read-outs in sparse field mode

\section{RADIATION TESTS}

Fig. 10 shows the set-up for testing the detector with $\gamma$-rays from a ${ }^{241} \mathrm{Am}$ source, which has a peak at $59 \mathrm{keV}$. Fig. 11 shows several examples of single event read-outs in sparse field mode. Figs. 12 and 13 show the spectrum measured from the ${ }^{241} \mathrm{Am}$ source in non-sparse mode and sparse-field mode respectively. The energy reported on the plot is the sum of a cluster of pixels. In non-sparse mode, the peak is close to $59 \mathrm{keV}$ as expected. The sparse-field data shows a peak at $45 \mathrm{keV}$ instead of $59 \mathrm{keV}$. This is probably because those pixels that received only a small share of the charge did not reach the triggering threshold of the sparse field readout, so the total energy recorded was less that the total deposited by the $\gamma$-ray. This performance may meet the requirements of an imaging application, but if not it could be corrected by automatically reading out the pixels adjacent those that trigger.

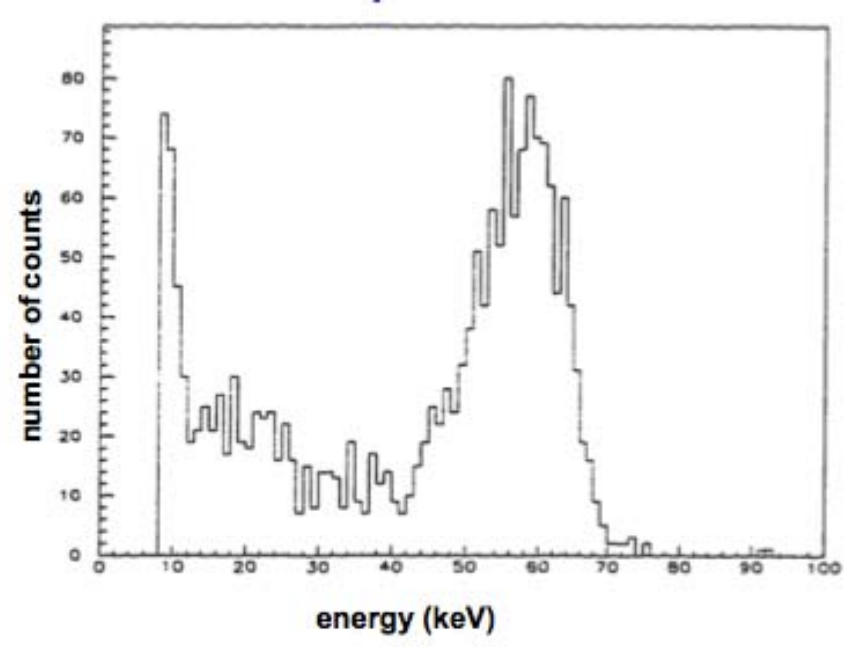

Fig. 12. ${ }^{241} \mathrm{AM}$ spectrum measured with the pixel detector in nonsparse field mode. The measured peak is $59 \mathrm{keV}$, as expected.

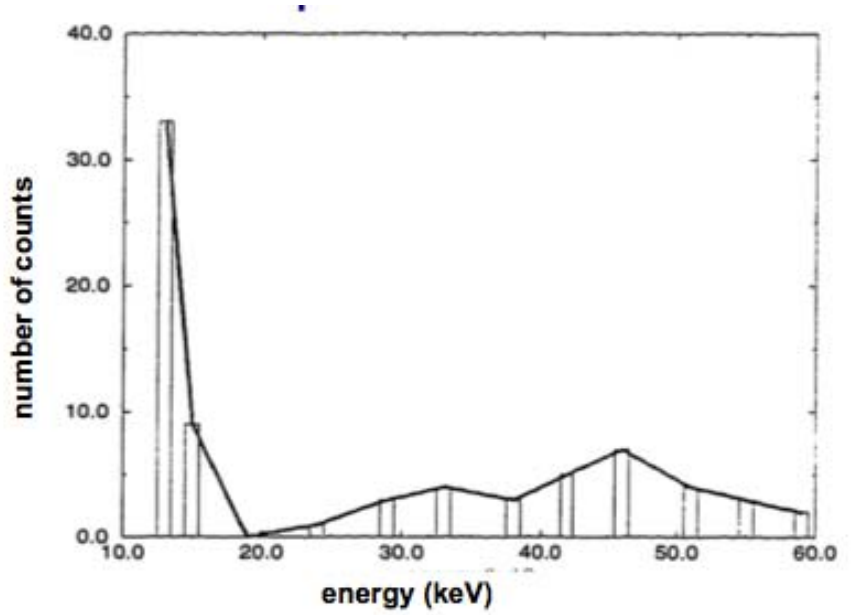

Fig. 13. ${ }^{241} \mathrm{AM}$ spectrum measured with the pixel detector in sparse field mode. The measured peak is $45 \mathrm{keV}$, due to the sparse field mode triggering set-up.

\section{CONCLUSION}

We have developed and tested a second generation monolithic pixel detector, with full CMOS circuits built directly in the high-resistivity bulk silicon. A vertical high voltage junction termination structure was implemented to reduce yield loss caused by double sided wafer processing. CMOS circuits are fully functional, including sparse field readout. High minority carrier lifetime was maintained in the substrate after the full process. Radiation tests demonstrated the feasibility of our approach for particle physics or x-ray imaging applications.

\section{REFERENCES}

[1] Snoeys, W.; Plummer, J.D.; Parker, S.; Kenney, C., "PIN detector arrays and integrated readout circuitry on high-resistivity float-zone silicon," IEEE Transactions on Electron Devices, vol.41, no.6, pp.903-912, Jun 1994

[2] Kenney, C. J.; Parker, S. I.; Peterson, V. Z.; Snoeys, W. J.; Plummer, J. D.; Aw, C. H. "A prototype monolithic pixel detector," Nuclear Instruments and Methods in Physics Research Section A: Accelerators, Spectrometers, Detectors and Associated Equipment, Volume 342, Issue 1, 15 March 1994, Pages 59-77 
[3] Segal, J.D.; Kenney, C.J.; Aw, C.H.; Parker, S.I.; Vilkelis, G.; Iwanczyk, J.S.; Patt, B.E.; Plummer, J., "A vertical high voltage termination structure for high-resistivity silicon detectors," Transactions on Nuclear Science, IEEE, vol.45, no.3, pp.364-369, Jun 1998

[4] Aw, C. H., CMOS Circuits for Integrated Particle Detection and Imaging Arrays, Ph. D. Thesis, Stanford University, 1996. 